\title{
TENDANCES ORIGINALES DANS L'EMPLOI DE CERTAINS ÉLÉMENTS DU LATIN SCIENTIFIQUE ET DE CHANCELLERIE Ȧ LA BASSE ÉPOQUE, CHEZ CLAUDIEN MAMERTUS
}

In memoriam optimae matris, Dominae

Ottiliae Iordache.

Presbytérien à Vienne (en Gaule), dans la moitie du V-e siècle, Claudien Mamertus est bien connu par ses préoccupations de philosophe et d'orateur.

Ses ouvrages en prose conservés (il s'agit des ouvrages sûrs de Claudien Mamertus: De statu animae, en trois livres, et des deux Epistulae, d'apres l'édition d'Auguste $G$. Engelbrecht ${ }^{1}$ ) ont en général un style soigné, souvent emphatique, propre à l'éloquence et à la poésie. L'influence de Cicéron, de Lucrèce, de Virgile et surtout d'Apulée, mais aussi de nombreux autres écrivains contemporains ou bien éloignés y est assez évidente. À ce propos, une caractérisation correcte du vocabulaire de Claudien Mamertus se trouve dans A. Ebert, Geschichte der christlichlateinischen Literatur: „Das Werk (De statu animae) offenbart sich auch in der Kühnheit, womit Claudian aus dem Sprachschatz der fernen Vorzeit wie der Gegenwart schöpft, allerdings mit Verzicht auf Eleganz des Ausdrucks.“2. Voici d'ailleurs les propres mots de Claudien Mamertus (dans sa lettre au rhéteur Sapaudus) au sujet des modèles stylistiques conseillés à son époque: „Naeuius et Plautus tibi ad elegantiam, Cato ad grauitatem, Varro ad peritiam, Gracchus ad acrimoniam, Chrysippus ad disciplinam, Fronto ad pompam, Cicero ad eloquentiam capessendam usui sint... illi ergo reuentilandi memoriaeque mandandi sunt, de quibus isti potuere proficere, quos miramur."

Cependant, bien que destinés aux catégories de gens cultivés, les ouvrages de Claudien Mamertus comportent de nombreux éléments de latin vulgaire, notamment dans les domaines de la syntaxe et du vocabulaire. Rappelons en ce sens (et ce à titre de dette contractée par Claudien Mamertus à l'endroit du latin vivant de son

1 Dans la collection „Corpus Scriptorum Ecclesiasticorum Latinorum“, vol. XI, Vienne, 1885 (édition anastatique: New York, Johnson, 1966, XLIX). A propos de la paternité des ouvrages, voir la Préface d'Auguste Engelbrecht de l'édition citée, p. 48-49.

2 op. cit., Leipzig, 1874, p. 452. Pour d'autres précisions sur le style de Claudien Mamertus, voir Aug. Engelbrecht, Untersuchungen über die Sprache des Claudianus Mamertus, dans „Sitzungsberichte der kaiserlichen Akademie der Wissenschaften in Wien“, Vienne, 1885, pp. 431-32; ibid., pp. $435-36$ etc. 
époque) la construction de l'interrogative indirecte avec l'indicatif, l'emploi fréquent de la conjonction quia causale, l'usage des locutions in quantum et in tantum.

Nous allons nous limiter, dans ce qui suit, à l'examen de l'emploi que fait Claudien Mamertus de l'adverbe tenus et de ses composés ${ }^{3}$. Précisons d'abord que tenus autant que sa famille comptent pour des mots importants dans le style scientifique et de chancellerie, de la Basse Latinité. Deux de ses composés, hactenus et serotinus, fréquents également dans le latin vulgaire, se sont conservés dans les langues romanes. Cela étant, l'étude de l'usage de tenus et de sa famille nous aide à mieux définir la latinité de Claudien Mamertus, en tant qu'écrivain et ecclésiastique. ${ }^{4}$

Prenons, pour commencer, la postposition TENVs, qui apparaît dans quatre exemples accompagnant un substantif à l'ablatif, selon la règle classique. Dans deux cas, tenus indique un SENS SPATIAL CONCRET: „jusqu'à “ (voir Stat. an., p. 144, 17 - II, 12: „aere tenus... est"; ibid., p. 176, 1 - III, 11: "latere tenus adstrepuerit". Cfr Virg., Aen., X, 210: „laterum tenus“). Dans d'autres cas, tenus comporte un SENS FIGURE,. INSTRUMENTAL-RESTRICTIF: „pas plus loin que“, „seulement d'après..." (emploi rare et plutôt prétentieux de cette postposition ${ }^{5}$ ). Voir Claudien Mamertus, Stat. an., p. 99, 3 - I, 27: „aliquibus (quaestionibus) superficie tenus adtactis“; ibid., p. 117, 1 - II, 5: „uerbo tenus“ (ce dernier syntagme est d'ailleurs copié sur Cicéron, Leg., III, 14).

Dans deux autres cas, tenus èst employé avec l'accusatif, construction fautive sur le modèle de $a d$, usque ad et usque. Le premier exemple de ce genre est postclassique, voir Valère Flaccus, I, 538, la construction avec l'accusatif demeurant d'ailleurs rare tout au long de la latinité ${ }^{6}$. Voici les exemples de Claudien Mamertus: a) Stat. an., p. 73, 6 - I, 21: „labrum tenus prolabitur“ (Cfr Lucrèce: „labrorum tenus“, Rer. nat., I, 940; le même exemple chez Lucrèce, IV, 15), b) Stat. an., p. 95, 1

3 Nous utilisons l'édition d'Auguste Engelbrecht, citée dans la note 1.

4 Il nous faut préciser que, dans l'étude, remarquable par ailleurs, d'Aug. Engelbrecht, Untersuchungen über die Sprache des Claudianus Mamertus (op. cit.), ne sont abordées que certaines innovations sur le plan de la formation de mots (tels eotenus, huccinetenus, adeotenus, itatenus) et cela d'une manière plus qu'incomplète (voir pp. 520-21). D'autres composés n'y sont que mentionnés (tels eatenus, hactenus, nullatenus, ullatenus, aliquatenus) - op. cit., p. 521 - sans qu'il y ait nulle référence au contexte, à la valeur sémantique, syntaxique et stylistique, à leur fréquence chez Claudien Mamertus et chez d'autres auteurs de l'époque, voire aux raisons ayant conduit à l'usage plus ou moins large de tenus ou de tel autre compośe chez un auteur, dans un courant ou une époque. La conjonction quatenus, aux emplois divers autant qu'intéressants chez notre auteur, n'y est même pas mentionnée.

Ajoutons encore que l'Index des mots qui clôt l'edition (citée note 1) d'Aug. Engelbrecht est incomplet. Pour l'adverbe ullatenus on indique deux exemples seulement au lieu de trois, etc.

Précisons aussi que les composés de tenus, sauf la conjonction quatenus, composés et formations appartenant à divers auteurs et époques, N'ONT ÉTÉ TRAITÉS QUE DE MANIËRE FORT SPORADIQUE ET INSATISFAISANTE DANS LES DIVERS TRAITÉS DE GRAMMAIRE ET DANS LES DICTIONNAIRES. C'est précisément ces lacunes que nous tâchons de combler par nos études portant sur l'usage de tenus et de ses composés chez Cicéron, Jordanès et divers auteurs de la basse époque.

5 Voir R. Kühner - C. Stegmann, Ausführliche Grammatik der tateinischen Sprache, Hannovre, 1971, II - 1, p. 517, par. 96, point 2, c.

6 Voir J. B. Hofmann - A. Szantyr, Lateinische Grammatik, II (München, 1965), p. 2681; R. Kühner - C. Stegmann, op. cit., II - 1, p. $517^{4}$, par $96,3^{\circ}$. 
- I, 26: „aurem tenus meat“. Dans ces exemples, tenus indique le sens spatial concert ,jusqu'à“. D'ailleurs cela ressemble plutôt à une règle valant tout le long de la latinité et qui veut que tenus accompagnant un accusatif n'ait d'autre sens que spatiall ${ }^{7}$. En tout cas, les deux exemples où tenus est construit avec l'accusatif se trouvent dans le premier livre de De statu animae.

Tenus en postposition apparaît donc six fois, en tout, dans De statu animae (alors qu'il fait défaut dans Epistulae).

L'emploi de tenus témoigne de l'aspiration de l'écrivain à un style élevé. En outre, six exemples c'est déjà beaucoup, par rapport aux auteurs classiques et tardifs (à comparer, naturellement, en termes absolus, ou relatifs, suivant l'étendue de chaque oeuvre concernée).

Il est à remarquer que Cicéron lui-même use fort rarement de cette postposition: quatre exemples seulement dans tous ses ouvrages ${ }^{8}$. Quant à Lucrèce, il n'emploie tenus que deux fois, et chaque fois dans la même expression: „labrorum tenus“ (voir les exemples cités ci-dessus) ${ }^{9}$. Chez César, tenus n'apparâtt point ${ }^{10}$, alors que chez Quintillien on le rencontre une seule fois ${ }^{11}$. Bon nombre d'auteurs de l'époque tardive l'emploient rarement ou fort rarement, tels Lactance, Optace de Milève, Orose, Cassien etc., alors que chez bien d'autres, tel Turranius Rufin, tenus fait complètement défaut.

Pour clore ce paragraphe consacré à la postposition tenus, rappelons que celleci indique parfois un sens figuré, instrumental - ce qui témoigne de la préciosité de l'auteur en question.

Tenus apparaît encore dans une autre hypostase, à savoir celle où il accompagne un adverbe. En voici un exemple dans De statu animae: „Huccine tenus est humani uisus animi?" (p. 173, 20 - III, 11). L'effet stylistique du syntagme se trouve d'ailleurs renforcé par la formation expressive, à consonne géminée, huccine ${ }^{12}$.

Un autre exemple du même genre apparaît toujours dans De statu animae: „...id est dicere: adeo tenus non est corpus anima, ut sit imago diuina.“ (p. 141, 910 , ou II, 10).

7 Cfr. J. B. Hofmann - A. Szantyr, op. cit., II, p. $268^{1}$.

8 Pour ce qui est de la fréquence et des valeurs de tenus chez Cicéron, voir notre ouvrage El uso del adverbio „quatenus" en las obras de Cicéron, dans „Helmantica“, vol. XXXVII (Salamanque, 1986), no 114, p. 12.

9 Voir Joh. Paulson, Index Lucretianus (Goetheburg - Leipzig, 1926. 2-éd.), p. 160.

10 Voir R. Kühner - C. Stegmann, A usführliche Grammatik der lateinischen Sprache, op. cit., II - 1, p.517, par. $96,2^{\circ}$.

11 Voir Ed. Bonnellus, Lexicon Quintilianeum (Hildesheim, 1962), p. 894.

12 Chez Claudien Mamertus on rencontre également haeccine - voir p. 48, 3. 
Les expressions sont, sans doute, dans les deux cas pléonastiques, car $h u c$, autant qu'adeo (notamment ce dernier) présentaient en clair le sens figure „à ce point“, à un tel point".

Les formules de ce genre sont fréquentes à la basse époque dans le latin vulgaire (mais aussi chez les auteurs cultivés, influencés par le latin vulgaire), voir, par exemple, l'emploi de la locution usque hactenus chez Jordanès (Romana, 84 et Getica, 273), ou bien Itinerarium Antonini Placentini, p. 188; 17, ibid., p. 189, 8 (édition „C.S.E.L.“, vol. XXXIX, Leipzig, 1898) ${ }^{13}$; voir encore la locution hactenus huc usque dans Servius, Aen., VI, 62, etc. ${ }^{14}$.

Naturellement, pour rendre le sens „jusqu'à ce point“, Claudien Mamertus pouvait toujours se servir des adverbes anciens: huc, eo, adeo, cependant, les formations en question ont, par rapport aux adverbes anciens (mono-, dis- et trisyllabiques) l'avantage non seulement d'une précision sémantique évidente, mais encore d'une expressivité artistique supérieure. fois.

Rappelons que huccine tenus, autant que adeo tenus apparaissent une seule

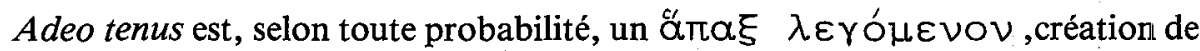
Claudien Mamertus ${ }^{15}$. La nouvelle création suit, naturellement, le modèle de eo usque. Huccine tenus est elle aussi une innovation de Claudien Mamertus, et qui demeure une formation isolée, utilisée seulement par cet auteur ${ }^{16}$. Elle est formée sur le modèle de huc usque et hoc usque ${ }^{17}$. Il n'est point exclu que Claudien Mamertus ait tenté de créer une série de syntagmes: adeo tenus, huccine tenus, sur le modèle de la série qui existait déjà: adeo usque, huc usque, hoc usque.

On se demande pourtant, en toute légitimité, pourquoi Claudien Mamertus s'attache à l'emploi de huccine tenus et de adeo tenus, alors que formations telles que eatenus ${ }^{18}$ et eo usque ${ }^{19}$ lui étaient bien familières? C'est, sans doute, par souci d'un style varié, Claudien Mamertus étant un inlassable chercheur de mots et d'expressions hautement suggestifs. D'autre part, une formation nouvelle (notamment avec tenus) était de nature à toucher davantage les lecteurs (ou les auditeurs).

13 Pour d'autres exemples de usque hactenus à la basse époque, voir Thesaurus linguae Latinae, VI - 3, fasc. 15 (Leipzig, Teubner, 1938), p. 2749, 80-84.

14 Voir Thesaurus linguae Latinae, VI - 3, fasc. 17 (Leipzig, 1942), p. 3071, 58-59.

15 Voir K. Georges - H. Georges, Ausführliches lateinisch-deutsches Handwörterbuch, I (Hannover Leipzig, 1913; édition anastatique: Basel, 1962), p.114. Voir aussi Aug. Engelbrecht, Untersuchungen über die Sprache des Claudianus Mamertus, op. cit., p. 477.

16 Voir Thesaurus linguae Latinae, VI - 3, fasc. 17, op.cit., p. 3073, 82-83.

17 Pour huc usque et hoc usque, voir Thesaurus linguae Latinae, VI - 3, fasc. 17, op. cit., p. 3072, 27 sqq. et p. 3074,4 sqq.

18 Pour eatenus, voir le propos qui suit immédiatement.

19 Pour eo usque, voir les exemples suivants chez Claudien Mamertus: Stat. an., p. 123, 3-4; ibid., p. $134,10-12 ;$ p. 136, 12-13; p. 161, 6-8; p.187, 17 (donc, cinq exemples). 
Voir, par ailleurs, l'emploi assez particulier de adeo tenus: au début d'une phrase qui frappe avec la force d'une sentence (voir l'exemple cité à la page précédente).

Il est à remarquer à ce propos que, pour le sens de „jusqu'à ce point“, on voit apparaître chez Claudien Mamertus d'autres formations nouvelles aussi: eotenus et itatenus (avec la variante ita tenus). Parmi ses innovations, un puissant relief stylistique semble comporter les formations de deux mots (où tenus ne fait pas corps avec l'adverbe antérieur). C'est pourquoi Claudien Mamertus crée et utilise, plus ou moins fréquemment, adeo tenus, huccine tenus et ita tenus. Cfr la graphie quando quidem - p. 44, 13, ou I, 6 (De statu animae).

Nous allons aborder maintenant les composés proprement dits de tenus, rappelant d'abord le groupe formé de EATENVS et EOTENVS. Eatenus et eotenus n'apparaissent qu'avec le sens figuré: „á tel point“, „tellement“.

Eatenus est assez fréquent chez Claudien Mamertus: on en compte dix exemples. D'ailleurs il y est deux fois plus frequent que eo usque (dont on trouve cinq exemples, voir note 19), la locution qui, chez Claudien Mamertus, fait principalement concurrence à eatenus pour le sens de ,jusqu'à tel point“" (car itatenus apparaît parfois avec le sens „ainsi“). D'ailleurs eo usque est bien répandu chez les auteurs tardifs $^{20}$ et il s'est conservé en logoudorien ${ }^{21}$.

On rencontre surtout eatenus en corrélation avec ut consécutif (dont quatre exemples, dans Stat. an., p. 63, 3-5, ou I, 17; ibid.., p. 85, 26 - p. 86, 1, ou I, 24; p. 123, 7-10, ou II, 7; p. 175, 16-17, ou III, 11). Eatenus apparaît ensuite en corrélation avec ut comparatif (deux exemples, dans De statu animae: p. 32,16-19, ou I, 3 et p. 115, 18-19, ou II, 5), avec quoad usque, avec le sens "dans la mesure où" (un seul exemple, Stat. an., p. 135, 7-9, ou II, 9) et enfin, avec si (un exemple, Stat. an., p. 47, 18-19, ou I, 8). Il est rare que eatenus apparaisse tout seul, sans être en corrélation avec une conjonction de subordination (voir Stat. an., 113, 9, ou II, 4; ibid., p. 141, 14, ou 10).

Certaines corrélations de Claudien Mamertus semblent être inspirées de Cicéron, à savoir: „eatenus - ut consécutif“ (Cicéron, Opt. gen., 23), ou bien „eatenus — quoad“ (Cicéron, Leg., I, 14; ad Q. fr., I, 1, 11). ${ }^{22}$

Eotenus n'apparaît que deux fois et seulement en corrélation avec ut consécutif. Les deux exemples se trouvent dans De statu animae:

20. Quant à la fréquence de eo usque à la basse époque, voir Thesaurus linguae Latinae, VII - 2, fasc. 4 (Leipzig, 1967), p. 488, 69 sqq.

21 Voir Wilh. Meyer - Lübke, Romanisches etymologisches Wörterbuch, Heidelberg, 1935, no. 2877.

22 Voir Thesaurus linguae Latinae, vol. V - 2 (Leipzig, 1910), p. 2, 51-52; ibid., p. 3, 4-6. 
1. „... eotenus inplicatur ${ }^{23}$ errore, $u t$ tamquam absens sibi se quaerat...“, p. 84,14 $-\mathrm{I}, 24$

2. „... eotenus religio conclamata est, $u t .$. scientiae fructum capessat...", p. 122, $8-11$, ou II, 7 .

Si eatenus est déjà attesté chez Cicéron ${ }^{24}$ et apparaît fréquemment à la basse époque ${ }^{25}$, eotenus est une création de Claudien Mamertus, étant d'un EMPLOI EXTRÊMEMENT RARE CHEZ LES AUTEURS POSTÉRIEURS ${ }^{26}$.

Eotenus apparaît chez Claudien Mamertus sur le modèle de eatenus et même de eo usque. Eotenus est bien peu utilisé par rapport à eatenus, étant cinq fois plus rare que ce dernier. En outre, Ĉlaudien Mamertus ne l'emploie qu'en corrélation avec ut consécutif.

Mais pourquoi Claudien Mamertus a-t-il recours à eatenus et eotenus? Sans doute, pour gagner en preécision sémantique, autant qu'en expressivité artistique.

En plus, il est à remarquer que, déjà lourds d'une force stylistique spéciale, ces adverbes bénéficient de positions privilégiées au niveau de la proposition ou de la phrase. Ainsi, eatenus est placé d'habitude au début de la proposition principale, ou au moins avant le verbe principal. Pour la position au début de la proposition principale, voir Stat. an., p.63, 3-5: „..., eatenus inest corpori, ut, sicut aqua utrem, minore sui parte minores partes eius inpleat ${ }^{27}$ et maiore maiores..."; voir aussi Stat. an., p. 135, 7-9.

Il y a un exemple où eatenus est souligné au point de vue sémantique et stylistique par l'adverbe equidem, et cela dans une phrase faisant office de conclusion à la suite de démonstrations antérieures: „Eatenus equidem ratio ponderis eo quod pendi potest cessante non interit et numerus finito numerabili non finitur." (Stat. an., p.113, 9-11).

Pour ce qui est de la position de eotenus dans la phrase et dans sa propre proposition voir les exemples ci-dessus.

Nous concluons ce paragraphe en précisant que les diverses formations de Claudien Mamertus significant „jusqu'à ce point“, étrangères à la l'usage classique,

23 inplicatur pour implicatur. Conserver le préfixe in- sous sa forme de base est un souci constant chez Claudien Mamertus (voir inplicabere - p. 199, 5, ou -Ep. I; inplebat - p. 162, 12, ou Stat. an., III, 6; inluminabile - p. 103, 19, ou Stat. an., II, 2, etc.).

24 Voir Thesaurus linguae Latinae, V - 2, op.cit., p. 2, 27 etc.

25 Voir Thesaurus linguae Latinae, V - 2, op. cit., p.3, 41 sqq.; ibid., p. 3, 59 sqq. etc.

26 Un seul exemple dans Jordanès, Getica, 30, et qui est, d'ailleurs, écrit en deux mots: eo tenus (d'après l'édition de Th. Mommsen, dans „Monumenta Germaniae historica“, V - 1, Hannovre, 1882; édition anastatique - Berlin, 1961). Au sujet des deux exemples de Claudien Mamertus et de l'unique exemple de eo tenus dans Jordanès, voir aussi Thesaurus linguae Latinae, $\mathrm{V}-2$, op. cit., p.651.

27 pour inpleat, voir note 23 . 
finissent par exclure totalement les adverbes $h u c$ et eo. Quant à l'adverbe adeo, celui-ci n'apparaît que dans un seul exemple (voir Stat. an., p. 54, 4).

Nombreuses et utilisées alternativement, les formations significant ,jusqu'à tel point", étrangères à l'usage classique, confèrent au texte un caractère fort varié. C'est dans les pages 122 et 123 de De statu animae qu'apparaissent eotenus (p. 122, 8), eo usque (p.123, 3) et eatenus (p. 123, 8).

Un autre composé de tenus, présent chez Claudien Mamertus, c'est ITATENVS. Innovation du même Claudien Mamertus, itatenus est relativement fréquent, il apparaît cinq fois. Il indique les sens suivants: „jusqu'à ce point“, „tellement", „ainsi“, „exactement ainsi“.

Itatenus est employé tantôt comme corrélatif de $u t$ (ou $u t t^{28}$ ) consécutif, tantôt comme corrélatif de sicut (ou sicuti) comparatif.

Tous les exemples où apparaissent itatenus se trouvent dans De statu animae. Itatenus - corrélatif pour la proposition consécutive apparaît trois fois (p. 140, $13-14 ;$ p. 143, 15-19; p.171, 3-4), seulement dans les livres II et III, alors que itatenus - corrélatif pour la proposition comparative $\mathrm{n}$ 'apparaît que deux fois ( $\mathrm{p}$. $149,14-17$; p. 151, 21-22), dans le chapitre 12 du deuxième livre.

La graphie de itatenus est parfois ita tenus, par exemple dans les deux phrases où cet adverbe sert de corrélatif pour sicut comparatif. Voir, par exemple: „Ac per hoc ita tenus corporeum caelum... terra dicitur, sicut aquarum et aeris corpora... terra dicuntur...“ (Stat. an., p. 149, 14-17, ou II, 12). On y remarque le sens de „exactement ainsi“.

Parmi les exemples où itatenus est corrélatif pour ut consécutif on compte un cas où celui-là est écrit en deux mots: ita tenus, alors que dans les autres cas tenus fait corps avec ita. Ecrit en deux mots et corrélatif de ut consécutif, ita tenus apparaît en fait en début de chapitre, assumant alors un rôle stylistique particulier: „Sana quippe catholicae fidei doctrina ita tenus... a mediis ad summa conscendit, $u t$ ... in tertium caelum Pauli pedissequos rapiat..." (livre II, chap. 12 - p.143, 15-19).

Ita tenus, écrit en deux mots, apparaît donc trois fois.

Cependant, itatenus, en un seul mot, ne manque point d'expressivité artistique. Voici son emploi dans une principale d'exhortation de De statu animae: „... duoque

28 Des graphies archaïsantes apparaissent fréquemment chez Claudien Mamertus, voir uti pour $u t$ à la page 140,$14 ;$ p. 185, 5 ; voir ueluti pour uelut - p. 109, 1; 159,13;185, 6; 205, 13 etc.; sicuti pour sicut - p. 121,$2 ; 166,7 ; 178,9 ; 178,21 ; 184,6$ etc. 
ista (spiritus et anima) itatenus uniantur, uti sint et sensu et uerbo indiscriminabilia..." (II, 10, ou p. 140, 13-14).

Itatenus (ou ita tenus) est formé sur le modèle de illatenus (présent chez Marc Aurèle et Apulée ${ }^{29}$, ce dernier étant d'ailleurs l'un des principaux modèles de Claudien Mamertus) et de illactenus (employé par Aulu-Gelle) ${ }^{30}$, mais également sur le modèle de la série nullatenus et ullatenus (voir, pour ces derniers, notre propos qui suit immédiatement). D'ailleurs, compte tenu de la graphie de illatenus, nullatenus etc., nous sommes portée à croire que notre auteur a conçu itatenus comme un composé, plutôt que comme une formation en deux mots.. Et, c'est pour des raisons de style que Claudien Mamertus se plaît parfois à l'ècrire en deux mots (cfr. chez Claudien Mamertus, la graphie: quando quidem).

Sans doute, l'emploi de itatenus est-il une tentative pour éviter les adverbes et les locutions ordinaires: adeo, tantum, in tantum (ce dernier, vulgaire), ita, sic, lesquels étaient fort nécessaires dans un ouvrage d'éloquence théologique (à savoir de théologie dogmatique).

Signalons que itatenus est relativement fréquent chez Claudien Mamertus, par rapport à l'étendue de l'ouvrage De statu animae. Il y est même plus fréquent que d'autres formations en -tenus de son groupe d'adverbes composés indiquant le mode, tels que: nullatenus et ullatenus, bien que ces derniers soient plus anciens et assez répandus à l'epoque tardive.

Claudien Mamertus a vraisemblablement voulu imposer aux langages scientifique, ecclésiastique, juridique et administratif aussi bien itatenus que d'autres formations adverbiales ou substantives.

Cela étant, itatenus demeure une formation prétentieuse, en fait une complication inutile du langage, qui n'a été reprise par aucun autre écrivain ${ }^{31}$.

L'insuccès qui frappe itatenus tient principalement au fait que le latin vulgaire disposait d'adverbes et de locutions adverbiales bien vigoureux (voir, à ce titre, tantum et in tantum, hoc modo, isto modo) concurremment avec l'adverbe monosyllabique sic, lesquels s'imposeront aux auteurs de la Basse Latinité (Claudien Mamertus y compris).

Peu fréquents chez Claudien Mamertus, NVLLATENVS et vLlatenvs y apparaissent, le premier quatre fois et le second, trois fois.

29 Voir Thesaurus linguae Latinae, VII - 1, fasc. 3 (Leipzig, 1936), p. 338, 56-60; voir aussi K. Georges - H. Georges, Ausführliches lateinisch-deutsches Handwörterbuch, II (Leipzig, 1918), p. 44

30 Voir Thesaurus linguae Latinae, VII -1 , fasc. 3, op. cit., p. 336, 4-6. Voir aussi J. B. Hofmann A. Szantyr, Lateinische Grammatik, II, op. cit., p. $641^{2}$.

31 Voir Thesaurus linguae Latinae, VII -2 , fasc. 4 (Leipzig, Teubner, 1967), p. 532, 19-24, voir aussi Aug. Engelbrecht, Untersuchungen, op. cit., p. 479. 
Vllatenus, composé plus récent de tenus (par rapport à nullatenus) et rarement employé à la basse époque (toujours par rapport à nullatenus) ${ }^{32}$, n'apparaît que dans le premier livre de De statu animae (voir I; 4 - p. 36, 26; I, 21 - p. 78, 6-7; I, 25 - p. 92, 15-16), alors que les occurrences de nullatenus se trouvent dans les premier et deuxième livres et dans l'Epilogue de l'ouvrage De statu animae (voir I, 8 p. 48, 2-3; I, 14 - p. 58, 2; II, 10 - p. 139, 19; Epilog. - p. 195, 2-3).

Nullatenus présente chez Claudien Mamertus le sens usuel du bas latin, à savoir: „de nulle manière“, "nullement“. Quant à ullatenus, il apparaît d'habitude dans des propositions negatives, selon l'usage élégant des grands auteurs ecclésiastiques et des personnalités politiques de la basse époque, voir, à titre d'exemples: ,,ipsam uero castam non dici ullatenus castitatem. " (p. 92, 15-16); voir, également, l'exemple de la page 78, 6-7. Il y a cependant une occurrence de ullatenus en proposition affirmative (p. 36, 26).

Vllatenus, autant que nullatenus remplissent un rôle stylistique majeur dans les passages en question (voir l'occurrence de ullatenus ci-dessus, p. 92, 15-16), où apparaît une adnomination rhétorique ${ }^{33}$. Nullatenus apparaît dans le titre même d'un chapitre $(I, 14)$, conférant à celui-ci un ton solennel: „Nullatenus spiritualia corporeis oculis uideri posse."

Nullatenus et ullatenus demeurent cependant rares chez Claudien Mamertus. D'ailleurs ces composés n'apparaissent point dans les Lettres de notre écrivain, ce qui révèle une fois encore leur caractère prétentieux, artificiel.

Tout enclin qu'il soit à la préciosité, Claudien Mamertus a pourtant recours, plus d'une fois, pour les sens en question, à d'autres adverbes ou locutions adverbiales. C'est ainsi que nullatenus subit la concurence de nullo modo (Stat. an., p. 178, 16) et surtout de nequaquam (Stat. an., p. 22, 11 etc. etc.). Vllatenus se trouve supplantée par quo pacto (Stat. an., p. 25, 7 et 115, 2) et aliquo pacto (p. 28, 3), quoquo pacto (Stat an., p. 89, 3) et quoquo modo (Stat. an., 111, 19; ibid., 178, 15) et, enfin, par quodam modo (Stat. an., 37, 7; ibid., p. 81, 5; p. 83, 1; p. 98, 6).

Les autres composés de tenus présents chez Claudien Mamertus sont: HACTENVS et PROTINVS.

Hactenus connaît quatre occurences, qu'on trouve toutes dans De statu animae (p.127, 18-19, ou II, 7; p. 133, 8-9, ou II, 9; p. 137, 14, ou II, 9; p. 172, 12-13, ou III, 11). A la différence d'autres auteurs tardifs ${ }^{34}$, Claudien Mamertus l'écrit toujours correctement avec aspirée.

32 Voir K. Georges - H. Georges, Ausführliches lateinisch-deutsches Handwörterbuch, II, op. cit., p. 3284; ibid., II, p. 1212; voir également J. B. Hofmann, Lateinische Umgangssprache, Heidelberg, 1926, par 77, p. 81. Voir aussi l'Index des mots des oeuvres de Cassien, dans „C. S. E. L.“, XVII ${ }^{\text {, }}$ Prague - Vienne - Leipzig, 1888 etc.

33 L'adnomination est bien fréquente chez Claudien Mamertus. Voir, á ce sujet, l'Index des mots de l'édition d'Auguste Engelbrecht, op. cit., p. 215.

34 Sur huit exemples de hactenus dans les oeuvres de Jordanès y en a trois où hactenus apparaît sans aspirée (voir Romana, 84 et 196 et Getica, 273). 
Dans tous ces exemples, hactenus indique un sens spatial-temporel: „jusqu'ici — jusqu'à présent (j'ai parlé de..., il a apporté des arguments sur...)“, à savoir: „Hactenus quidem de animi incorporea atque inlocali uisione disserui...“, Stat. an.., p. 172, 12-13.

Dans certains cas, hactenus s'avère d'une force stylistique très marquée, se trouvant placé en début de proposition ou même de chapitre. Pour ce qui est de hactenus (renforcé par quidem) placé en début de chapitre, voir l'exemple cité tout à 1'heure: Stat. an., p. 172, 12-13. Voici un autre exemple intéressant: „Sed hactenus satis Ambrosius, uel solus profecto sit satis, faxim tamen et aliis testificandi locum. " (Stat. an., p. 133, 8-9), où l'on peut remarquer l'absence du verbe-copule argumentari. Claudien Mamertus semble s'inspirer, à propos de cet usage, de Cicéron, qui emploie hactenus même comme interjection jouant le rôle d'un verbe (voir Cicéron, Diu., II, 76: „nunc hactenus.“ — „maintenant, en voilà assez!“; voir également Cicéron, Brut., 52: „sed de Graecis hactenus. “ — „mais en voilà assez sur les Grecs.").

Si le sens „jusqu'ici - jusqu'à preśent“ de hactenus est bien fréquent à la basse époque $^{35}$, ce qui frappe c'est sa rareté chez Claudien Mamertus. L'explication réside sans doute dans la rare nécessité du sens „jusqu'à présent“ dans les oeuvres de Claudien Mamertus.

On peut constater également que sens le „jusqu'à tel point“ de hactenus, bien fréquent dans le bas latin ${ }^{36}$, y fait complètement défaut. Cela s'explique partiellement, chez Claudien Mamertus, par l'existence de plusieurs formations et composés nouveaux en -tenus, et cela en plus du vieil eatenus et du syntagme eo usque signifiant „à tel point“.

Cependant, l'usage peu fréquent de hactenus, par rapport à eatenus, vient à l'encontre de l'usage qu'en font les grands prosateurs latins ${ }^{37}$ et pourrait être consi-

35 Pour la fréquence de ce sens à l'epoque postclassique, voir F. Gaffiot, Dictionnaire illustré latin-français, Paris, 1934, p. 733; voir aussi K. Georges - H. Georges, Ausführliches lateinisch-deutsches Handwörterbuch, I, op. cit., p. 3001. Pour la fréquence de ce sens à l'époque tardive, voir Itinerarium Antonini Placentini, dans "C.S.E.L. “, vol. XXXIX (Leipzig, 1898), p. 189, 8; voir aussi Thesaurus linguae Latinae, VI - 3, fasc. 15 (Leipzig, 1938), p. 2749, 66 sqq.

D'ailleurs hactenus s'est conservé en logoudorien (voir Wilh. Meyer-Lübke, R.E.W., op. cit., no. 3967).

36 Le sens "jusqu'à tel point" de hactenus est présent chez de nombreux auteurs tardifs, voir Epistulae imperatorum, pontificum, aliorum ab an. 367 usque ad an. 553, dans "C.S.E.L.", XXXV - 1 (Prague-Vienne-Leipzig, 1895), p. 57, 6. Voir aussi Jordanès, Romana, 196. Voir encore Thesaurus linguae Latinae, VI -3 , fasc. 15, op. cit., p. 2751, 80 sqq.

37 Nous rappelons que Cicéron emploie, dans ses oeuvres, le composé hactenus 60 fois, tandis que pour eatenus il n'existe que 5 attestations (Sur la fréquence de hactenus et eatenus chez Cicéron voir notre étude:: El uso del adverbio "quatenus"en las obras de Cicerón, op. cit., p. 13). Quintilien utilise hactenus huit fois et eatenus, une seule fois (voir Ed. Bonnellus, Lexicon Quintilianeum, op. cit., p. 376 et 269.) Pline le Jeune et Apulée ignorent l'emploi de eatenus, mais ils utilisent hactenus (voir X. J. J. van Ooteghem, Index de Pline le Jeune, Namur, p. 365; voir Wil. Abbott Oldfather - H. Vernon Canter - B. E. Perry, Index Apuleianus, Middletown, 1934, p. 179). 
déré comme une tendance novatrice de Claudien Mamertus, à savoir de réduire les sens des deux adverbes (hactenus et eatenus) et de procéder à leur spécialisation sémantique.

Protinus, un composé fort ancien de tenus (dejà attesté chez Plaute ${ }^{38}$ ) connaît quatre occurrences, dont trois dans De statu animae et une seule dans Estitulae. Claudien Mamertus l'emploie avec le sens d'immédiatement", fort courrant à la basse époque.

L'exemple figurant dans Epistulae est particulièrement important, du fait qu'il témoigne une fois de plus de la large diffusion de l'adverbe protinus dans le langage des hommes cultivés de l'epoque tardive.

Quant à la position de protinus dans la proposition, il est à remarquer qu'il y bénéficie d'une place privilégiée, comme l'indique précisément cet exemple: „O si mihi de his loqui tecum... proueniret, ...nonnulla tibi de meo iure concederem neque protinus de homine quaererem..." (Stat. an., p. 107, 13-15, ou II, 3).

Voici aussi l'exemple tiré des Epistulae: „Quoniam si peccabis ultra reticendo, ego protinus ulciscar scribendo.“ (Ep., I — p. 199, 20-21).

Rappelons encore que protinus, autant que le composé hactenus (examiné plus haut), formaient de parfaits dactyles, fait d'importance majeure pour le rhytme de la proposition en question.

L'usage peu fréquent de protinus chez Claudien Mamertus s'explique par la non-nécessité du sens „immédiatement“. L'adverbe classique statim, tout comme le vulgaire $\operatorname{mox}$ sont extrêmement rares chez: Claudien Mamertus (chacun n'apparaissant qu'une seule fois: voir p. 178, ligne 26 pour statim et p. 181 , ligne 14 pour mox). Par rapport à ces derniers, protinus est donc relativement frequent.

Le dernier composé de tenus présent chez Claudien Mamertus c'est la conjonction QVATENVS. De par ses nombreuses occurrences (par rapport à d'autres composés) et ses différentes hypostases sémantiques-syntaxiques, quatenus est le composé le plus important de la famille de tenus à apparaître dans les ouvrages de Claudien Mamertus et des autres écrivains de la basse époque.

Aussitôt la question se pose: Pourquoi Claudien Mamertus a-t-il recours à la conjonction quatenus? C'est que le style scientifique et de chancellerie de l'époque tardive connaissait, sans doute, l'usage de quatenus, usage favorisé par certains facteurs: premièrement quatenus présentait l'avantage d'une plus grande précision sémantique (y compris celui d'un corps phonetique plus large), par rapport à beau-

38 Voir K. Georges - H. Georges, Ausführliches lateinisch-deutsches Handwörterbuch, II, op. cit., p. 2036; voir G. Lodge, Lexicon Plautinum, Hildesheim, 1962, vol. II, p. 398. 
coup d'autres conjonctions annciennes, surchargées de valeurs, usées et dépourves d'expressivité (tout d'abord par rapport à ut, cum, quoniam, qua et quam). D'autre part, quatenus, conjonction plus récente et ayant un nombre de valeurs sémantiques assez limité (du moins à l'époque classique et postclassique) n'exigeait pas de corrélatif.

Quatenus était, parfois, préférée à d'autres conjonctions en raison aussi du caractère solennel qu'elle conférait à des passages contenant des exhortations. En voici un chez Claudien Mamertus: „...neque in societate eius (corporis), nisi quatenus necesse est, animum dimittamus. " (Stat. an., p. 126, 25-26, ou II, 7). Le fragment est d'ailleurs une traduction d'après Phaedon de Socrate (même préférence pour quatenus face à d'autres conjonctions chez Cicéron, dans ses traductions des philosophes grecs - voir Cicéron, Tim., 13, 46). Rappelons aussi que la conjonction quatenus formait un parfait dactyle.

Des raisons stylistiques d'importance mineure commandent également le choix de la conjonction quatenus: si, par exemple, tel passage emploie plusieurs fois $u t$ comparatif, consécutif et final, on a alors recours à quatenus (pour introduire une subordonnée comparative, finale ou consécutive), afin d'éviter la répétition de la conjonction $u t$ (tel est le cas d'un passage de De statu animae, p. 117, lignes $1-8$, II, 5). D'autre fois, par l'emploi de la conjonction quatenus, on évite la répétition de la conjonction quantum.

Quatenus n'est employé que dans De statu animae (alors que d'autres écrivains ecclésiastiques, tels les évêques Fauste de Riez et Magnus Félix Ennode, à qui quatenus est familier, l'emploient couramment même dans les Épitres ${ }^{39}$ ). Il apparaît quatre fois dans le troisième livre, deux fois dans le deuxième et une seule fois dans le premier (avec une valeur habituelle). Ses différentes hypostases sémantico- syntaxiques apparaissant chez Claudien Mamertus se présentent comme suit:

a) propositions interrogatives indirectes - quatre exemples (dont trois construits avec le subjonctif: p.159, 18-19, ou III, 3; p. 177, 15-16, ou III, 12; p. 179, 10, ou III, 13; et un autre avec l'indicatif: p. 69, 1, ou I, 19);

b) proposition restrictive avec l'indicatif - un seul exemple, p. 126, 25-26, ou II, 7 (voir l'exemple cité plus haut);

c) proposition comparative-concessive, avec l'indicatif - un exemple, p. 182, 12-13, ou III, 14.

d) proposition finale, avec le subjonctif - un seul exemple, p. 117, 5-8, ou II, 5.

L'influence de Cicéron apparaît évidente dans l'emploi de quatenus introdluisant des interrogatives indirectes et des propositions restrictives. Pour ce qui est des interrogatives indirectes, voir Cicéron, De orat., II, 87, 355: „Itaque soli, qui menno-

39 Pour Fauste de Riez, voir l'Index des mots dans „C.S.E.L.“, vol. XXI, Prague-Vienne-Leipzig, 1891; pour Ennode, voir l'Index des mots, dans “C.S.E.L.“, vol. VI, Vienne, 1882. 
ria uigent, sciunt, quid et quatenus et quomodo dicturi sint...", par rapport à Claudien Mamertus, p. 159, 18-19: „nulla quaestionis difficultate iactabere, quatenus anima corpore posito inlocaliter esse possit in mundo...“.

Pour une subordonnée restrictive, voir Cicéron, Ep.ad Fam., I, 2, 1: „Quatenus de religione dicebat, cui quidem rei iam obsisti non poterat, Bibulo adsensum est...“, par rapport à la restrictive de Claudien Mamertus, p. 126, 25-26: „...neque in societate eius (corporis), nisi quatenus necesse est, animum dimittamus. "Dans le passage en question de Claudien Mamertus, p. 126, lignes 22-28, quatenus restrictif alterne avec in quantum restrictif. D'origine vulgaire, cette locution présentait l'avantage d'une grande précision sémantique. Claudien Mamertus emploie, dans ce fragment, in quantum, pour éviter la répétition de quatenus - rappelons que le passage entier représente une traduction d'après Phaedon de Socrate.

Si quatenus introduisant des propositions interrogatives indirectes et restrictives est dejà présent chez Cicéron, son emploi final ne semble attesté qu'au I-er siècle $n . e .^{40}$, alors que son emploi comparatif-concessif est, selon toute probabilité, une innovation de Claudien Mamertus ${ }^{41}$. A la différence de son emploi final, l'emploi concessif de quatenus demeure fort sporadique tout au long de la latinité.

Quatenus comparatif-concessif apparaît, chez Claudien Mamertus, dans l'exemple auivant: „En quatenus de localitate animae disputauisti, in hoc tamen temet ignauiae non arcesso." (Stat. an., p. 182, 12-13, ou III, 14).

Voyons aussi l'exemple de quatenus final: „Quatenus igitur numeri ueritatem in anima reperiamus humana, primum debet ipsa sibimet ${ }^{\mathbf{4 2}}$ actitandi rationem pro uirtutum parilitate concinere..." (Stat. an., p. 117, 5-8, ou II, 5).

Il en ressort que les exemples de quatenus comparatif-concessif et quatenus fi-

40 En ce qui concerne le moment de l'apparition en latin de la subordonnée finale introduite par quatenus, celui-ci est plus ancien que ne le conçoit Ed. Wölfflin et, après lui, toute une série de chercheurs (voir Ed. Wölfflin, Quatenus, dans "Archiv für lateinische Lexicographie und Grammatik“, vol. V 1, Leipzig, 1888, p. 408: "Der finale Gebrauch von quatenus beginnt in der Litteratur mit Tertullian und in der Jurisprudenz gleichzeitig mit Paulus und Ulpian". Voir aussi H. Goelzer, Le latin de Saint Avit, Paris, 1909, p. 350; Fried. Stolz - J. H. Schmalz, Lateinische Gramatik, II - 2, op. cit., p. $656^{1}$, par. 358.)

La plus ancienne attestation de la construction "quatenus + subjonctif" ayant une nuance finale dont nous disposons est d'un type quelque peu différent: il s'agit d'une proposition sujet à nuance finale - voir Sextus Iulius Frontinus, De aquaed. comm., 7, 2-à la fin du I-er siècle. Mais, à en juger par cet exemple, nous considérons que quatenus final proprement-dit était déjà employé au I-er siècle ap. J. Chr.

41 Dans son étude portant sur quatenus (op. cit.), Ed. Wölfflin ne signale chez aucun des auteurs latins l'usage concessif de cette conjonction.

42 sibimet - le pronom réfléchi et, d'autres fois, le pronom personnel, renforcés de la particule -met sont bien fréquents chez Claudien Mamertus, en accord avec le style emphatique de l'ouvrage De Staí animae. Voir aussi l'exemple cité plus haut: temet (p. 182,13). Voici, sur une seule page (page 127) les formes: semet (ligne 6), de nouveau semet (ligne 13), egomet (ligne 18). 
nal apparaissent, chez Claudien Mamertus, uniquement dans les deuxième et troisième livres.

Force nous est cependant de préciser que les hypostases sémantiquessyntaxiques courantes chez Claudien Mamertus sont les mêmes qui sont familières à Cicéron: 1 . la proposition interrogative indirecte; 2 . la proposition comparative.

Par l'emploi varié qu'il fait de la conjonction quatenus et, en outre, par l'usage nouveau, comparatif-concessif, qu'il y confere, Claudien Mamertus se distingue de la plupart des écrivains de la Basse latinité, chez lesquels prédomine, voire est exclusif, l'usage de cette conjonction avec le subjonctif (suppléant ut final, ut consécutif, ou bien ut complétif et subjectif, de nuance finale ou consécutive), comme c'est le cas d'Avit, d'Ennode, de Cassiodore, de Jordanès.

Pour ce qui est des propositions interrogatives indirectes introduites par quatenus chez Claudien Mamertus, il faut signaler un écart par rapport aux normes classiques, à savoir la construction du verbe avec l'indicatif (Stat. an., p. 69, 1 - I, 20: „quatenus anima nec quantitati subiacet disseramus...").

Il nous faut préciser que les exemples de ce genre (quatenus introduisant une interrogative indirecte avec l'indicatif) sont rares tout au long de la latinité. En tant qu'élément du style scientifique et de chancellerie, quatenus est, généralment, fort soigneusement construit.

L'apparition de l'indicatif chez Claudien Mamertus est due à l'influence du latin vulgaire. L'indicatif est autrement relativement fréquent - dans De statu animae - dans l'interrogative indirecte simple (après quid, quam, quot etc.) et même dans l'interrogative indirecte double.

Quatenus occupe, en général, une position privilégiée dans les passages en question. A part le fait qu'il se trouve, généralement, en premier lieu dans la proposition respective, sa subordonnée précède d'ordinaire la proposition régissante. Voici la proposition comparative-concessive: „En quatenus de localitate animae disputauis$t i$, in hoc tamen temet ignauiae non arcesso." (Stat. an., p.182, 12-13). Ajoutons encore que cette phrase est le début même d'un paragraphe du chapitre 14 du livre III.

La position de quatenus dans la phrase est bien souvent analogue à celle de quantum, importante conjonction vulgaire de la basse époque. Voir, à ce propos, De statu animae, Epil.: „Ergo duobus modis quantum in hac uita datur omnis rei habetur notitia.“ (p.192, 7-8).

La proposition comparative connaît une toute autre position, lorsque la proposition régissante comporte le corrélatif itatenus. Voir, en ce sens, De statu animae, p. $151,21-22$ : „...ita tenus nesciat, sicuti cum corpore an sine corpore sit raptus ignorat." 
Voici aussi la position de certaines interrogatives indirectes introduites par quatenus: „...quatenus dixeris quaero...“(Stat an., p.179, 10); voir également De statu animae, p. 69, 1 (l'exemple cité plus haut, construit avec l'indicatif). Voir, à titre comparatif, les interrogatives indirectes introduites par quid chez Claudien Mamertus. Quid (avec le sens de „quoi?") est un vocable très affectionné par le latin vulgaire de l'époque tardive. Par exemple: „...quid sit sciamus...“ (Stat. an., Epil. — p. 193, 14).

L'unique proposition finale introduite par quatenus, chez Claudien Mamertus, se trouve toujours placée avant la proposition principale (voir l'exemple cité - Stat. an., p. 117, 5-8).

Avant de clore ce paragraphe, nous tenons à préciser que, par rapport à l'usage qu'en font d'autres auteurs de la basse époque et compte tenu des dimensions peu étendues des ouvrages de notre auteur, la conjonction quatenus est fréquente chez Claudien Mamertus, qui en use dans sept cas.

On voit apparaître aussi, dans l'ouvrage De statu animae, un composé de quatenus à savoir ALIQVATENVS, qui y figure une seule fois, dans le passage suivant: „Igitur si inlocalitas animae uel aliquatenus adparuit, ... quatenus anima nec quantitati subiacet disseramus..." (p. 68, 23 - p. 69, 1, ou I, 19).

Aliquatenus présente le sens, d'ailleurs usuel à la basse époque, de: „jusqu'à un certain point". Quant à l'adverbe $u e l$, celui-ci supplée de manière erronée saltim ${ }^{43}$.

Déja présent chez Columelle et Pomponius Méla ${ }^{44}$, aliquatenus connaît un usage fréquent à la basse époque ${ }^{45}$. Son emploi peu fréquent chez Claudien Mamertus s'explique par la concurrence que lui fait la locution quodam modo (pour quodam modo, voir des exemples p. 81,$5 ;$ p. 83,1 etc.).

En résume, l'ouvrage De statu animae présente bien des composés de tenus. On y trouve également un composé de quatenus, à savoir aliquatenus. Dans Epistulae n'apparaît que protinus et une seule fois. Certains composés sont des innovations de Claudien Mamertus, tels que eotenus et itatenus; les syntagmes huccine tenus? et adeo tenus sont eux aussi des innovations. Les formations de deux mots du type adeo tenus semblent avoir un relief stylistique d'une force toute particulière. De là précisément la fréquence relativement élevée de telles formations chez Claudien Mamertus.

43 uel apparaît assez souvent, chez Claudien Mamertus, avec le sens de saltim (pour cette particularité de uel voir Index uerborum et locutionum de l'édition d'Aug. Engelbrecht, op. cit., p. 259). Vel pour saltim apparaît aussi chez d'autres auteurs de la basse époque (voir Jordanès, Romana, 2).

44 Voir Thesaurus linguae Latinae, vol. I (Leipzig, Teubner, 1900), p. 1605, 78 sqq.; ibid., p. 1606, 1 sqq.; voir aussi K. Georges - H. Georges, Ausführliches lateinisch-deutsches Handwörterbuch, vol. I, op. cit., p. 314 .

45 Voir Thesaurus linguae Latinae, vol. I, op. cit., p. 1606, 21-41. 
Certains composés y sont fréquents: eatenus (dont on compte dix exemples), quatenus (sept exemples), alors que d'autres y sont moins employés: hactenus (quatre exemples), protinus (quatre exemples), nullatenus (quatre exemples) etc.

La présence, chez Claudien Mamertus, de la postposition tenus rend compte de l'aspiration de l'écrivain à un style élevé. En plus, les six exemples qu'on trouve chez lui font nombre par rapport aux auteurs classiques et tardifs.

Il y a, chez Claudien Mamertus, des influences évidentes de Cicéron, telles que: l'emploi de tenus au sens figuré, instrumental-restrictif, l'emploi fréquent de la conjonction quatenus, certaines hypostases sintaxiques de quatenus (celle d'introduire des interrogatives indirectes, puis des propositions comparatives) etc.

D'autres innovations propres à Claudien Mamertus résident, par exemple, dans l'adjonction d'une hypostase sémantico-syntaxique à la conjonction quatenus, laquelle introduit, de ce fait, une proposition comparative-concessive.

On trouve aussi des erreurs, bien que peu nombreuses, par rapport aux normes du latin cultivé: tenus construit avec l'accusatif, emploi de ullatenus en proposition affirmative, présence de l'indicatif dans une interrogative indirecte. Signalons, en outre, un fait bien intéressant: tous ces écartes apparaissent dans le premier livre de De statu animae. Quant aux deuxième et troisième livres, on y trouve un style prétentieux et compliqué à souhait, parfois inutilement (pour donner un exemple, toutes les propositions contenant itatenus ou ita tenus apparaissent dans les livres II et III).

L'emploi de tenus et de sa famille permet, sans doute, de mieux définir la latinité de Claudien Mamertus. On peut affirmer, à.juste raison, que l'auteur en question s'avère le plus intéressant de toute la basse époque quant à l'emploi de cette famille de mots. D'abord parce qu'il utilise la plupart des composés de tenus; ensuite, parce que leur fréquence est, d'ordinaire, élevée (par comparaison, naturellement, à d'autres auteurs de l'époque et aux dimensions de ses ouvrages et, aussi, compte tenu de la nécessité à laquelle-répondent certains sens). La postposition elle-même y est assez répandue.

Des tendances originales sont à déceler sous d'autres angles encore: la graphie de ita tenus (deux mots), nouvelles hypostases sémantico-syntaxiques pour certains composés: quatenus comparatif-concessif; ita tenus signifiant: „ainsi“, „exactement ainsi“, corrélatif de sicut comparatif; la réduction des sens du classique hactenus; la forte présence stylistique des formations en tenus au niveau de la proposition et, bien souvent, de la phrase.

Claudien Mamertus se distingue encore des autres auteurs de l'époque par le nombre élevé de formations nouvelles (d'un ou deux mots), lesquelles ne réussirent pas cependant à s'imposer aux prosateurs contemporains ou postérieurs. 
Quoi qu'il en soit, Claudien Mamertus reste l'un des plus actifs écrivains ecclésiastiques sur le plan des innovations lexicales et syntaxiques.

Les formations nouvelles conduisent, conjointement avec les anciennes, à la création de larges séries de synonymes, dont les termes different autant sous l'aspect de la précision sémantique que de l'expressivité artistique. Voici la série de synonymes pour le sens „jusqu'à tel point": huccine tenus, adeo tenus, eo usque, itatenus (avec la variante ita tenus), eatenus et eotenus. Ajoutons-y le vieil adverbe adeo.

L'emploi particulier de tenus et de sa famille, ainsi que d'autres familles de mots, témoignent, chez Claudien Mamertus, de la mise en jeu de toutes les ressources lexicales et syntaxiques, au profit de l'argumentation philosophique et de la délectation artistique.

Povzetek

\section{ZNANSTVENO IN ADMINISTRATIVNO IZRAZOSLOVJE V POZNI LATINSKI DOBI: SVOJSKOSTI V RABI KLAVDIJANA MAMERTA}

Kar zadeva uporabo izrazov iz družine TENUS je res mogoče trditi, da je Claudianus Mamertus najbolj zanimiv pisec pozne latinske dobe. Predvsem je tenus kot člen sestavljenk uporabljen dokaj pogosto; polleg tega je visoka tudi frekvenca sestavljenk s tenus. Zmeraj seveda primerjamo njegov jezik z jezikom drugih piscev pozne latinščine; obenem pa je treba upoštevati obsežnost njegovega opusa.

Zapostavljanje prislova tenus $v$ sestavljenkah je dokaj pogostno v delu De statu animae. Svojske težnje so vidne tudi sicer: grafija ita tenus (ločeno), drugačni pomeni nekaterih sestavljenk, kot ita tenus v pomenu 'tako', 'prav res tako', kar je korelativno k primerjalnemu sicut, ali quatenus v primerjalnokoncesivnem pomenu. Nadalje omejevanje pomenov klasičnega hactenus in stilistična vrednost sestavljenk v stavku ali celo v povedi.

Claudianus Mamertus je različen od drugih avtorjev pozne latinš̌čine tudi glede na visoko število novih skovank (eno- ali dvobesednih), ki se še niso uveljavile $v$ jeziku njegovih sodobnikov in tudi ne pri pozneǰ̌ih piscih. Kar zadeva semantične in sintaktične inovacije, je Mamertus sploh eden najbolj aktivnih cerkvenih piscev pozne dobe.

Način, kako je uporabljen tenus (in njegova pomenska družina), je lep primer, kako Mamertus uporablja vsa sredstva, leksikalna in skladenjska, za poglobljeno filozofsko dokazovanje, obenem pa za resnični umetniški užitek. 